\title{
Effect of hydrogen bonding interactions on the release mechanism of felodipine from nanodispersions with polyvinylpyrrolidone
}

\author{
Evangelos Karavas ${ }^{\text {a,b }}$, Georgios Ktistis ${ }^{\mathrm{a}, *}$, Aristotelis Xenakis ${ }^{\mathrm{b}}$, Emmanouel Georgarakis ${ }^{\text {a }}$ \\ a Department of Pharmaceutical Technology, School of Pharmacy, Aristotle University of Thessaloniki, Thessaloniki, Greece \\ ${ }^{\mathrm{b}}$ Institute of Biological Research and Biotechnology, National Hellenic Research Foundation, Athens, Greece
}

Received 1 July 2005; accepted in revised form 9 January 2006

\begin{abstract}
Solid dispersion systems are widely investigated for the dissolution enhancement of poorly water soluble drugs. Nevertheless, very limited commercial use has been achieved due to the poor predictability of such systems caused by the lack of a basic understanding of the dissolution optimization mechanism. In the present study an investigation of the release mechanism is performed for solid dispersion systems composed by polyvinylpyrrolidone (PVP) and felodipine (FEL), based on a correlation of their hydrophilicity with the intensity of interactions. The existing interactions were evaluated by using NMR and UV spectroscopy while molecular simulation techniques were also enabled. It was found that the interactions that take place correspond to the creation of hydrogen bonds. The correlation between the intensity of interactions and the concentration of PVP in the matrix showed a sigmoid function. The interactions are impressively increased for polymer concentration exceeding $75 \%(\mathrm{w} / \mathrm{w})$. This phenomenon was well explained by using the molecular simulation technique. A similar sigmoid pattern was found for the function between dissolution profiles and polymer concentration in the matrix, indicating that the intensity of interactions promotes the dissolution enhancement. Investigation of the solubility and the particle size distribution of FEL in the binary system appeared to have similar behaviour indicating that the interactions affect the release profile through these two factors. The hydrophilicity of PVP does not significantly affect this enhancement as the contact angle was found to be linear to PVP concentration. Microscopic observation of the dissolution behaviour showed that FEL remains in fine dispersion in aqueous solution, verifying the release mechanism.
\end{abstract}

(C) 2006 Elsevier B.V. All rights reserved.

Keywords: Felodipine; Solid dispersions; Nanodispersions; Interactions; Release mechanism

\section{Introduction}

The enhancement of the bioavailability of poorly water soluble drugs is one of the main targets of drug development during the last decades. Several techniques have been developed concerning the optimization of the dissolution rate of these drugs. Such methods include particle size reduction, solubilization, salt formation and preparation of solid dispersion systems [1].

\footnotetext{
* Corresponding author. Department of Pharmaceutical Technology, School of Pharmacy, Aristotle University of Thessaloniki, Thessaloniki 54124, Greece. Tel.: +30 2310 997653; fax: +30 2310997652.

E-mail address: ktistis@pharm.auth.gr (G. Ktistis).
}

Nevertheless, there are several disadvantages and limitations in the use of these techniques [2]. Specifically, the particle size reduction technique is practically limited regarding the minimum size, which could be achieved, while the production of very fine powders deteriorates their flow properties and wettability, while it advances the development of electrostatic forces, leading to problematic formulations. The solubilization techniques correspond to liquid preparations, which usually do not assure the patient's acceptability and normally appear to have poor stability. Salt formation is a complicated process, while it is not feasible for neutral compounds. Additionally, the dissolution enhancement is not always predictable due to the high correlation of the salt's solubility with the $\mathrm{pH}$ value which appears a high variability in the gastrointestinal tract. 
Solid dispersions incorporating poorly water soluble drugs into hydrophilic carriers were introduced by Sekiguchi and Obi since 1961 [3]. However, their commercial use is very limited due to their poor predictability. As referred to by Craig [4], in a specific review, poor predictability of solid dispersion is assigned to the lack of a basic understanding of their properties, especially their solid state structure and stability as well as of the dominant mechanisms that facilitate dissolution enhancement.

In our previous work [5] a treatment of felodipine (FEL) with polyvinylpyrrolidone (PVP) was investigated. The characterization of the system showed that they were amorphous nanodispersions, possibly based on the formation of a hydrogen bond between FEL and PVP. In that paper, we investigated the formation mechanism and the solid state characteristics of the systems including chemical and physical stability.

Nanodispersions are preferable drug delivery systems regarding the dissolution rate. Especially, concerning drug substances with low solubility and high permeability (like FEL and other dihydropyridines), these systems are optimal as recrystallization caused by the saturation of the gastrointestinal fluid is limited. Similar studies have also been performed in the past [6] for the dissolution enhancement of FEL, through solid dispersion systems with cellulose derivatives.

The preparation of these systems presupposes the presence of intermolecular interactions between the drug and the polymeric carrier in order to avoid drug agglomeration and recrystallization or formation of metastable forms. These interactions could be characterized as critical factors for the physicochemical properties of the resulting systems.

In the present study, an investigation of the possible mechanism for a dissolution enhancement is performed via its correlation to the existing drug-polymer interactions. Although several suggestions have been proposed, the mechanism by which the dissolution rate is improved, relative to the conventional dosage forms, is still not fully understood. A review by Chiou and Riegelman [7] indicates two possible mechanisms corresponding to the particle size reduction or reduced agglomerates and to increased solubility or dissolution rate of the drug. The first mechanism corresponds to a link between solid state structure and dissolution behaviour. Indicative examples of the second mechanism are the formation of soluble complexes between drug and carrier as it is well established for cyclodextrins. Additionally, physicochemical modification of the drug such as degree of crystallinity, polymorphic form, or amorphous state could be included in this category. Nevertheless, according to later studies the second mechanism appears to be more complicated. Corrigan $[8,9]$ found that the dissolution rate of the drug from the tested solid dispersion system was equivalent to the one of the polymer (PEG) hypothesizing a carrier-controlled mechanism. This hypothesis was supported by Dubois and Ford [10], who found that the dissolution rates of different drugs were similar, when the same carrier and manufacturing conditions were used. Later, Craig [4] found that the dissolution process was directly correlated with the molecular weight of the carrier (PEG), while Lloyd et al. [11] presented that in some cases the physical form of the drug is irrelevant to the release rate in the case of carrier-controlled mechanism. Nevertheless, parallel studies by other investigators [12] showed a linear function between the drug solubility and the dissolution rate, proposing that this behaviour should be referred to as drug-controlled dissolution.

In the present study, identification and characterization of the interactions between FEL and PVP is performed using NMR and UV spectroscopy techniques. Additionally, a correlation between the interactions' intensity and the hydrophilicity of the systems is investigated. It is well established that the presence of strong heteromolecular forces, especially hydrogen bonds, may differentiate the physicochemical characteristics of the initial molecules [13]. Due to this behaviour the dissolution rate and the bioavailability of these systems cannot be predicted as they are not proportionally relative to the drug:polymer ratio. The investigation of the correlation between the intensity of interactions and the hydrophilic properties of the systems is appropriate during formulation development in order to avoid the preparation of nonrepeatable and nonaccurate systems.

FEL is a dihydropyridine practically insoluble in water [14], but highly permeable, while its dissolution rate is the limiting factor for its bioavailability. Nevertheless, the dissolution profile of dihydropyridines is not always representative of their bioavailability due to the fact that several surfactants are used in the dissolution medium in order to achieve sink conditions. For this reason in the present study an extended investigation is performed regarding the effect of hydrogen bond on several characteristics as solubility, dissolution profile, contact angle, etc. This study, firstly, addresses the evaluation of the parameters affecting the release profile of FEL from its solid dispersions with PVP and secondly, the investigation of the exact mechanism of the dissolution enhancement, as well as the correlation between the effects of interactions and other representative parameters of the FEL hydrophilicity.

\section{Materials and methods}

\subsection{Materials}

Felodipine (FEL) with an assay of $99.9 \%$ was obtained from PCAS (Longjumeau, France). Polyvinylpyrrolidone (PVP) type Kollidon K30 with a molecular weight of 50,000-55,000 was obtained from BASF (Ludwigshafen, Germany). All the other materials and reagents were of analytical grade of purity.

\subsection{Preparation of the nanodispersions}

The method of dissolution in a common solvent was followed. The appropriate amounts of FEL and PVP were 
dissolved in ethanol absolute and sonicated. The solvent was then fully evaporated after exposure at $40^{\circ} \mathrm{C}$ for $48 \mathrm{~h}$ in an appropriate air ventilated dry oven (Memmert). Glass Petri dishes were used as trays. The created films were pulverized and sieved up to a particle size of 150-250 $\mu \mathrm{m}$. The following FEL:PVP ratios of 75:25, $50: 50,45: 55,40: 60,35: 65,30: 70,25: 75,20: 80,15: 85$, 10:90 and 5:95 (w/w) were prepared.

\section{3. ${ }^{1} H$ NMR spectroscopy}

The pure compounds (FEL-PVP) and the FEL:PVP systems of 10:90, 25:75, and 50:50 (w/w) were examined using nuclear magnetic resonance (NMR). The experiments were performed on an NMR spectrometer type BRUCKER DRX $400 \mathrm{MHz}$, while deuteronized chlorophorm $\mathrm{CDCl}_{3}$ was used as a solvent.

\section{4. $U V$-vis spectroscopy}

UV-vis absorption spectra were recorded on a Shimadzu UV-vis spectrophotometer model UV 1601. Different ratios of FEL:PVP were measured dissolved in ethanol absolute as it was the solvent for the preparation of the nanodispersions. The spectra were obtained in the $\lambda_{\mathrm{em}}=200-450 \mathrm{~nm}$ range. The maximum absorbance of FEL was measured at $362 \mathrm{~nm}$. All the solutions was corresponded to a FEL concentration of $0.05 \mathrm{mg} / \mathrm{ml}$, while relevant amounts of PVP were spiked in order to achieve the following FEL:PVP mass ratios of 90:10, 80:20, 70:30, 60:40, 50:50, 40:60, 30:70, 20:80, and 10:90 (w/w). The test was performed in triplicate while the RSD per sample was found to be less than $2 \%$.

\subsection{Solubility in water at $37^{\circ} \mathrm{C}$}

A dissolution apparatus II of USP (paddles) type DISTEK was used. Hard gelatin capsules containing samples of each system equivalent to $50 \mathrm{mg}$ of FEL were placed into the vessels into hard gelatin capsules under the conditions: $500 \mathrm{ml} \mathrm{H}_{2} \mathrm{O}, 50 \mathrm{rpm}, 37^{\circ} \mathrm{C}, 36 \mathrm{~h}$. The capsules were held by metallic nets at the bottom of the vessel in order to achieve repeatable hydrodynamic conditions. After $36 \mathrm{~h}$ a sample of $50 \mathrm{ml}$ per vessel was removed, filtered through $0.2 \mu \mathrm{m}$, and evaporated under vacuum on a rotary evaporator, BUCH Rotavapor R200 equipped with a vacuum controller model V-800 and a heating bath model B-490. The residues were dissolved in $5 \mathrm{ml}$ ethanol absolute and assayed using the HPLC method described in European pharmacopoeia. The analysis was performed on a type Shimadzu instrument model LC-20-10A, at $237 \mathrm{~nm}$ using a $\mathrm{C}_{18}$ column. The disintegration time of the hard gelatin capsules did not affect the results of the experiment as it was very rapid (less than $5 \mathrm{~min}$ ). The experiment was performed in triplicate, while the RSD per sample was found to be less than $1.5 \%$.

\subsection{Drug particle size evaluation}

The prepared solid dispersion systems were examined using a scanning electron microscope (SEM), type Jeol (JMS-840). For this examination films of solid dispersions fractured by liquid nitrogen were used. All the studied surfaces were coated with carbon black to avoid charging under the electron beam. The Martin diameters were measured for the dispersed particles of FEL, using a suitable digital ruler.

\subsection{Microscopic evaluation of the dissolution behaviour}

In order to investigate quantitatively the hydrophilicity of the systems, a dynamic microscopic method was developed. Specifically, microtablets of pure FEL and the FEL:PVP systems of 25:75 and 10:90 (w/w), with a diameter $2.97 \mathrm{~mm}$ and weight $5 \mathrm{mg}$, were prepared using a low compression force of $50 \mathrm{kp}$ for $5 \mathrm{~s}$. The tablets were placed in Petri dishes under a microscope type Olympus. The appropriate amount of purified water in order to cover the upper surface of the microtablets was added while microphotographs were taken for each sample. In order to compare the nanodispersions with the relative physical mixtures the appearance of a FEL:PVP physical mixture of 10:90 (w/w) was correlated with the relevant solid dispersion at a magnification of $100 \times$.

\subsection{Contact angle}

Cylindrical tablets of pure FEL and the nanodispersion systems were prepared with a diameter of $11 \mathrm{~mm}$ and weight of $300 \mathrm{mg}$. The compression force was $1000 \mathrm{kp}$ for $5 \mathrm{~s}$. The tablets were sprayed with purified water. After an application of one drop $(50 \mu \mathrm{l})$ of purified water on the upper surface of the tablets they were immediately photographed.

The contact angle was measured with a digital goniometer after magnification of the photographs. Pure FEL and the FEL:PVP systems of 75:25, 50:50, 25:75 and 10:90 $(\mathrm{w} / \mathrm{w})$ were measured. The experiment was performed in triplicate while the RSD between the measurements of identical samples was found to be less than $5 \%$.

\subsection{Release kinetics}

A modified dissolution apparatus II of USP (paddles) type DISTEK, equipped with a stationary disk in order to achieve hydrodynamic repeatability, was used. The test was performed at $37^{\circ} \mathrm{C}$ and $100 \mathrm{rpm}$, while $500 \mathrm{ml}$ phosphate buffer, $\mathrm{pH} 6.5$, containing polysorbate $20 \%$ of $2 \%$ $(\mathrm{w} / \mathrm{w})$ in order to achieve sink conditions was used. Samples were collected every $5 \mathrm{~min}$ and analyzed according to the HPLC method described above. Pure FEL and the FEL:PVP systems of 10:90, 15:85, 20:80, 30:70, 40:60, 50:50 and 75:25 (w/w) were evaluated. The test was 
performed in triplicate while the RSD was found to be less than $3 \%$.

\subsection{Dynamic particle size evaluation during dissolution}

The particle size distribution of the FEL nanoparticles during dissolution of solid dispersions was determined using a dynamic light scattering (DLS) process with a Zetasizer Nano Instrument (Malvern Instruments, Nano ZS, ZEN3600) operating with a 532-nm laser. A suitable amount of solid dispersion was immersed in purified water, corresponding to a concentration of $1 \%$, at $37^{\circ} \mathrm{C}$ and agitation at $200 \mathrm{rpm}$. Particle size formation of the samples was measured at different dissolution times.

\section{Results and discussion}

\subsection{Preparation of the nanodispersions}

It was found that the systems were amorphous dispersions of FEL in PVP matrix for polymer concentrations of over $50 \%(\mathrm{w} / \mathrm{w})$, while the FEL:PVP system of 75:25 $(\mathrm{w} / \mathrm{w})$ was found to be crystalline. The particle size distribution of FEL was found to be less than $500 \mathrm{~nm}$. The development of interactions between the carbonyl group of PVP and the amino group of FEL was identified as the reason for the optimal dispersion. The identification was performed by using FT-IR spectroscopy. These interactions lead also to a partial miscibility of the substances, which is the possible mechanism for the formation and stabilization of the glassy state. The resulted systems appeared to have enhanced dissolution and solid state stability, depending on polymer concentration in the matrix. Nevertheless, the exact mechanism which leads to the release enhancement was not fully investigated, at this stage, as FT-IR spectroscopy is not suitable for a quantitative evaluation of interactions. For this reason NMR and UV spectroscopy were also enabled in order to establish a correlation between the exact mechanism leading to the dissolution enhancement and the existing interactions.

\section{2. ${ }^{1}$ H NMR spectroscopy}

${ }^{1} \mathrm{H}$ NMR spectroscopy was used in order to identify the nature of interactions between FEL and PVP for the FEL:PVP systems of 50:50, 25:75 and 10:90 (w/w) and pure materials.

The use of $\mathrm{CDCl}_{3}$ as a solvent for the ${ }^{1} \mathrm{H}$ NMR experiments for the identification of the interactions between FEL and PVP was chosen in order to satisfy several purposes. Firstly, both the compounds are soluble in the above solvent. Secondly, and more important, $\mathrm{CDCl}_{3}$ is an aprotic solvent, which means that it will not exchange the $\mathrm{NH}$ proton of FEL, thus having the chemical shifting on the ${ }^{1} \mathrm{H}$ NMR responding to the one in the solid state. In addition $\mathrm{CDCl}_{3}$ as an aprotic solvent will not interact to the potential hydrogen bonding between FEL and PVP. Finally, the possibility of interactions from the presence of traces of water has been excluded by the use of dry $\mathrm{CDCl}_{3}(99.96 \%$ Aldrich).

A comparative presentation of pure FEL and the FEL:PVP systems of 25:75 and 10:90 (w/w) is shown in Fig. 1. The spectrum of the FEL:PVP system of 50:50 $(\mathrm{w} / \mathrm{w})$ is not presented as the basic peaks appear similar to the ones observed when pure FEL is examined.

Following analysis of the above spectra, resonance of the methyl in the carboxyl group is observed at $1.16 \mathrm{ppm}$ (triple peak integrated for three protons), resonance of the two methyl groups in the ortho position of the Nitrogen at $2.27 \mathrm{ppm}$ (two single peaks, each integrated for three protons) resonance of the methoxy group of the carbomethoxy group at $3.54 \mathrm{ppm}$ (single peak,

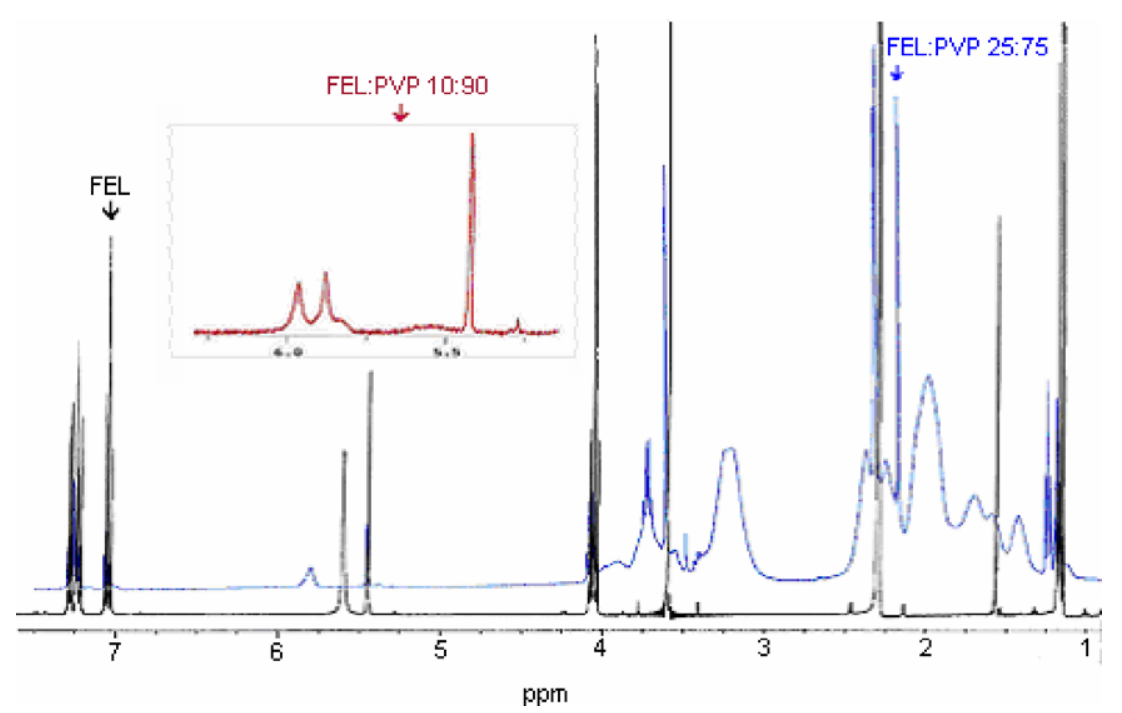

Fig. 1. Proton NMR spectra for pure FEL and the FEL:PVP system of 25:75 (w/w). The small picture (up and left) presents the amino group of FEL in the FEL:PVP system of 10:90 (w/w). 
integrated for three protons), and resonance of the methylene of the carboepoxy group at $4.09 \mathrm{ppm}$ (quadruple peak that integrated for two protons). Finally, the aromatic protons exhibit resonance at $7.02 \mathrm{ppm}$ (single triple, ortho-ortho junction that integrates for one proton), at $7.19 \mathrm{ppm}$ (single double-double, ortho-meta junction that integrates for one proton) and at $7.22 \mathrm{ppm}$ (for single double-double, ortho-meta junction that integrates for one proton). The proton that is located on the nitrogen atom (amino group of FEL) and exhibits resonance at $5.52 \mathrm{ppm}$ (single peak that integrates for one proton) is of great interest.

The proton spectra of FEL:PVP system of 50:50 (w/w) do not show any changes, but in the FEL:PVP system of 25:75 (w/w) the proton on the nitrogen atom appears to be vulnerable and exhibits resonance at $5.80 \mathrm{ppm}$. The same proton, in the proton spectrum of the 10:90 mixture, appears as double peak, one at $5.80 \mathrm{ppm}$ and one at $5.95 \mathrm{ppm}$.

From the above experiments it is observed that the increase of the PVP concentration solidifies the hydrogen bond between the proton and amino group of FEL and the carboxyl group of PVP. Especially, in the FEL:PVP system of 10:90 (w/w) two peaks are observed corresponding to the protons that have formed, one strong and a second weaker hydrogen bond with the PVP carbonyl group. Although the 50:50 (w/w) shows an alteration of the $>\mathrm{NH}$ using FTIR, the same does not happen when using ${ }^{1} \mathrm{H}$ NMR. This is due to the possible existence of a weak hydrogen bond in the dispersion system mentioned above, which is capable of altering the movement properties of the $\mathrm{N}-\mathrm{H}$ bond, but incapable of weakening the proton of the amino group.

It is clear that the intensity of interactions is very low in the FEL:PVP system of 50:50 (w/w), while the hydrogen bond becomes stronger for polymer concentrations above $75 \%(\mathrm{w} / \mathrm{w})$. In order to explain this phenomenon the molar ratios between FEL and PVP repeat unit in the systems were taken under consideration. As the molecular weights are 386.24 for FEL and 111.14 for PVP, the molar ratios in the investigated systems were calculated as presented in Table 1.

As shown in Fig. 2 the size of the PVP repeated unit is much smaller than that of the molecule of FEL. Specifically, the size of the molecule of the active substance corresponds to those of more than four-repeated units of the polymer. Taking into consideration Table 1 and Fig. 2 it can be concluded that up to a polymer concentration of

Table 1

FEL:PVP weight and molar ratios of investigated systems

\begin{tabular}{lll}
\hline Designation & Weight ratio (w/w) & Molar ratio \\
\hline 1 & $75: 25$ & $1: 1.16$ \\
2 & $50: 50$ & $1: 3.48$ \\
3 & $25: 75$ & $1: 10.43$ \\
4 & $10: 90$ & $1: 31.29$ \\
\hline
\end{tabular}

$50 \%(\mathrm{w} / \mathrm{w})(1: 3.48)$, the amount of PVP is inadequate to interact with the whole amount of FEL, resulting in a very weak hydrogen bond that cannot be identified by NMR spectroscopy. However, taking into consideration that the placement of PVP chains in any system is not straight but helicoid the required ratio between FEL:PVP rises up to 1:40 (molar ratio) in order for the interactions to be fully developed. According to the above it can be concluded that the intensity of hydrogen bond interactions is increased by increasing the polymer concentration in the systems. The appearance of two peaks corresponding to the protons of amino group at 5.80 and $5.95 \mathrm{ppm}$ for the FEL:PVP system of $10: 90(\mathrm{w} / \mathrm{w})$ indicates that two different kinds of interactions are present in the system. This behaviour could be explained by the partial miscibility identified in our previous work [5]. It was observed that in these systems, although they are immiscible, a quantity of FEL is present in PVP rich phase and the opposite. As there are two different distributions in the systems, there appear two different peaks in NMR spectroscopy. Specifically, for the FEL:PVP system of 10:90 (w/w) the amount of FEL in PVP rich phase was found to be about $15 \%(\mathrm{w} / \mathrm{w})$. Similar investigations have been performed in the past by other researchers. Hosono et al. [15] demonstrated complex formation between the amide group of PVP and the aromatic ring of Ajmaline while Van den Mooter et al. [16] suggested the absence of such interactions for Ketoconazole-PVP k25 systems, by using solution and solid state NMR techniques.

The findings of this study are in full compliance with the results of the FTIR analysis presented in our previous work [5].

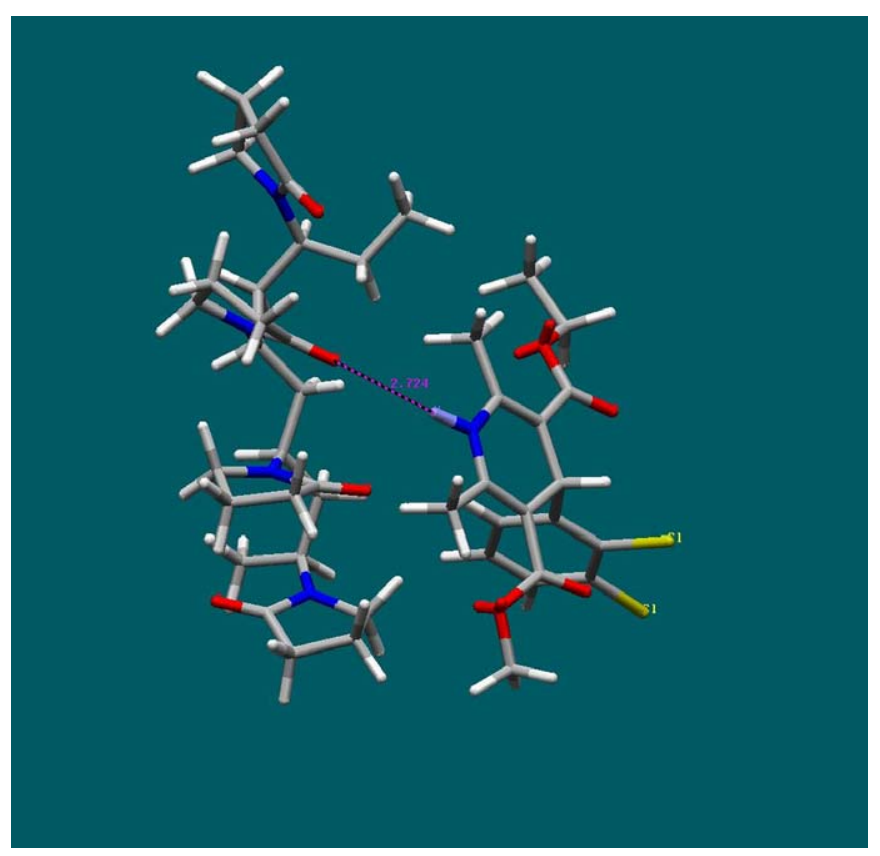

Fig. 2. Molecular simulation for the creation of the hydrogen bond between FEL (right) and PVP (left). 


\section{3. $U V$-vis spectroscopy}

A quantitative evaluation of the existing hydrogen bonding interactions was investigated using absorption UV-vis spectroscopic techniques. Different ratios from $10 \%$ to $90 \%(\mathrm{w} / \mathrm{w})$ polymer concentrations in the system were measured. As the solvent for the evaluation was identical (ethanol) with the one used for the preparation of the system, it could be said that the results correspond to the intensity of interactions during the formation of the nanodispersions. It was observed that upon the addition of PVP the absorption intensity increased, while no significant shift on the $\lambda_{\max }$ was observed. This increase was not due to any absorbance of PVP, as its spectrum did not deviate from the base line as shown in Fig. 3.

The characteristic increase of FEL absorption has to be attributed to the presence of interactions between polymer and drug. In this case, these interactions correspond to the presence of the hydrogen bond between the amino group of FEL and the carbonyl group of PVP as identified by NMR spectroscopy. Valero et al. [17] observed similar behaviour for the systems containing Naproxen-PVP and they attributed this behaviour to an ion-dipole interaction. Similar investigations using UV-vis or fluorescence emission spectra have also been performed by Matsumoto and Zografi [18] describing the complex of Indomethacin and PVP, while Velazquez et al. [19] observed spectroscopic modifications in the quantum yield of fluorescence of Naproxen $\mathrm{Na}$.

Although these techniques are widely used for the evaluation of interactions, the quantitative correlation between the polymer concentration on the systems and the appearance of interactions has not yet been properly investigated. In order to evaluate this correlation in the present study a normalized representative factor $(F)$ for the interaction intensity was calculated according to the equation:

$F=\frac{A_{\mathrm{S}}-A_{0}}{A_{0}}$

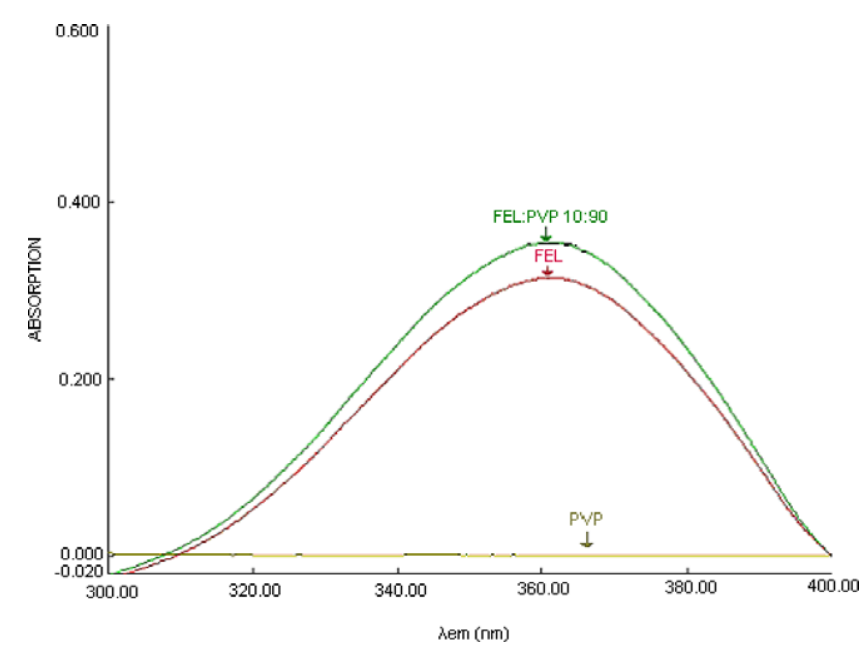

Fig. 3. Indicative UV spectra of the pure substances (FEL, PVP) and the FEL:PVP system of 10:90 (w/w). where $A_{\mathrm{S}}$ and $A_{0}$ represent the absorbance at maximum (362 nm) of the complex and free drug, respectively.

The function between interaction intensity $(F)$ and the polymer concentration in the system is presented in Fig. 4. The results are in full agreement with the observations by NMR spectroscopy. After partial evaluation of the curve it can be seen that an impressive increase of the slope is observed for polymer concentrations over $50 \%$ $(\mathrm{w} / \mathrm{w})$ (molar ratios more than 1:4). The examination of the increase of interaction identity is similar to the one given in the discussion of the results given by NMR spectroscopy, concluding that strong interactions take place for a polymer concentration over $50 \%(\mathrm{w} / \mathrm{w})$, while the maximum intensity is achieved in the area of polymer concentrations over $75 \%(\mathrm{w} / \mathrm{w})$.

\subsection{Solubility in water at $37^{\circ} \mathrm{C}$}

As the solubility behaviour is one of the factors affecting the dissolution rate, a correlation with the interactions that

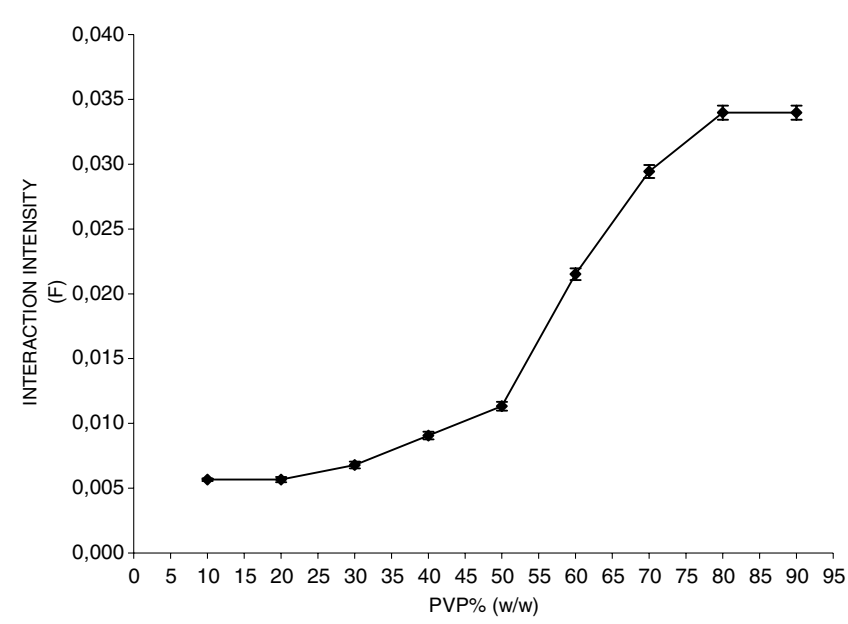

Fig. 4. Correlation between PVP concentration in the system and interaction intensity (F) evaluated by UV-vis spectroscopy.

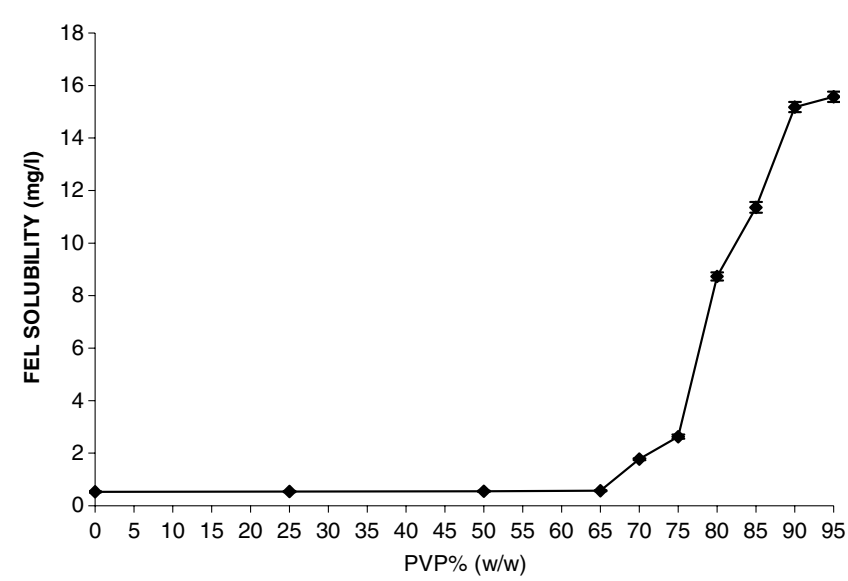

Fig. 5. Function between FEL solubility in water $\left(37^{\circ} \mathrm{C}\right)$ relative to the PVP concentration in the systems. 
take place and their effect on the dissolution rate is performed in the present study. According to the method published by Higuchi and Connors [20] the solubility of the drug substance is usually measured in aqueous solutions containing different concentrations of the polymer. In the present work the solubility at $37^{\circ} \mathrm{C}$ of the FEL:PVP systems containing PVP of $25 \%, 50 \%, 65 \%, 70 \%, 75 \%$, $80 \%, 85 \%, 90 \%$ and $95 \%(\mathrm{w} / \mathrm{w})$ was evaluated.

It is observed (Fig. 5) that the correlation between solubility and polymer concentration in the system follows similar sigmoid pattern with the intensity of the interactions presented in Fig. 4. In addition, no significant enhancement of the solubility is obtained up to a polymer concentration of $70-75 \%(w / w)$. For polymer concentrations over $75 \%$ $(w / w)$ an impressive increase of the slope is observed.

Comparing the two curves indicating interactions (Fig. 4) and solubility (Fig. 5) it can be seen that the increase of solubility shows a delay compared to the one of interaction intensity. Nevertheless, it is observed that the solubility enhancement is taking place for polymer concentrations over $75 \%(\mathrm{w} / \mathrm{w})$, where the interaction intensity appears to be the maximum. This observation is well correlated with the existing interactions. It has to be attributed to the formation of soluble complexes between the hydrophobic drug and the hydrophilic carrier, based on the identified hydrogen bond. Similar suggestions have been expressed by several researchers for the possible mechanism of the solubility enhancement of several drugs. Most of them observed linear regression between drug solubility and polymer concentration. Specifically, Sethia and Squillante [21] found a linear correlation between the solubility of carbamazepine and the concentration of PVP k25 and PVP k30. Similar behaviour was presented by Mura et al. [22] and Bettinetti and Mura [23] for NaproxenPVP, evaluating in parallel the presence of cyclodextrin systems. Mura et al. [24] investigating Naproxen-Chitosan systems found that the drug solubility was increased as the polymer concentration increased, and a type $\mathrm{A}_{\mathrm{N}}$, according to Higuchi and Connors equation was obtained. This behaviour was also attributed to the formation of a weak soluble complex between Chitosan and Naproxen. Solubility improvement was also observed by Daniel Mwambete [25], evaluating three Benzimidazole carbamate drugs.

In our case the relationship between polymer concentration and solubility is absolutely different and the systems exhibit two different behaviours at low and high concentrations of PVP. This is a strong indication that the hydrogen bonding interactions are the responsible mechanism for the solubility enhancement. However, the solubility of FEL is impressively enhanced in the area of concentrations where the interaction intensity is the maximum. In detail, as the solubility of pure FEL at $37^{\circ} \mathrm{C}$ was found to be $0.53 \mathrm{mg} / \mathrm{L}$, the binary system containing PVP of $90 \% \quad(\mathrm{w} / \mathrm{w})$ corresponds to a FEL solubility about 30 times higher. Similar behaviour, especially for FEL, has been observed by Kerč et al. [14]. Micronized FEL and PEG mixtures appear to have higher solubility $(3.00 \mathrm{mg} / \mathrm{L})$ than crystal- line FEL $(0.26 \mathrm{mg} / \mathrm{L})$. This solubility enhancement of coprecipitates was attributed to several factors including the particle size reduction, the interactions between PEG and FEL and the solubility effect of the hydrophilic carrier. According to our opinion, regarding the systems presented in this study, particle size reduction cannot affect the solubility, but only the dissolution kinetics as the systems were measured after $36 \mathrm{~h}$ stirring, while the capacity of PVP as a solubilizer is limited. According to the above, the basic mechanism for the solubility enhancement seems to be the intensity of the hydrogen bonding interactions between drug and polymer and the formation of a complex between FEL and PVP in aqueous media. Nevertheless, the particle size of the systems is also investigated.

\subsection{Drug particle size evaluation}

The particle size distribution of the drug substance was evaluated as it is strongly correlated to the surface exposed to the dissolution medium affecting through this mechanism the release kinetics of the drug substance.

As a representative factor for the investigation, the specific surface $\left(S_{\mathrm{u}}\right)$ of the particles was evaluated as it is correlated with the surface mean diameter of the FEL particles. As presented in Fig. 6 the particles are almost spherical, and the calculation of $S_{\mathrm{u}}$ was evaluated according to the equation:

$S_{\mathrm{u}}=\frac{6}{d_{32}}$

where the number 6 indicates the spherical shape while $d_{32}$ is the surface mean diameter given from the following equation:

$d_{32}=\frac{\sum n_{i} d_{i}^{3}}{\sum n_{i} d_{i}^{2}}$

where $d_{\mathrm{i}}$ is the measured Martin diameter.
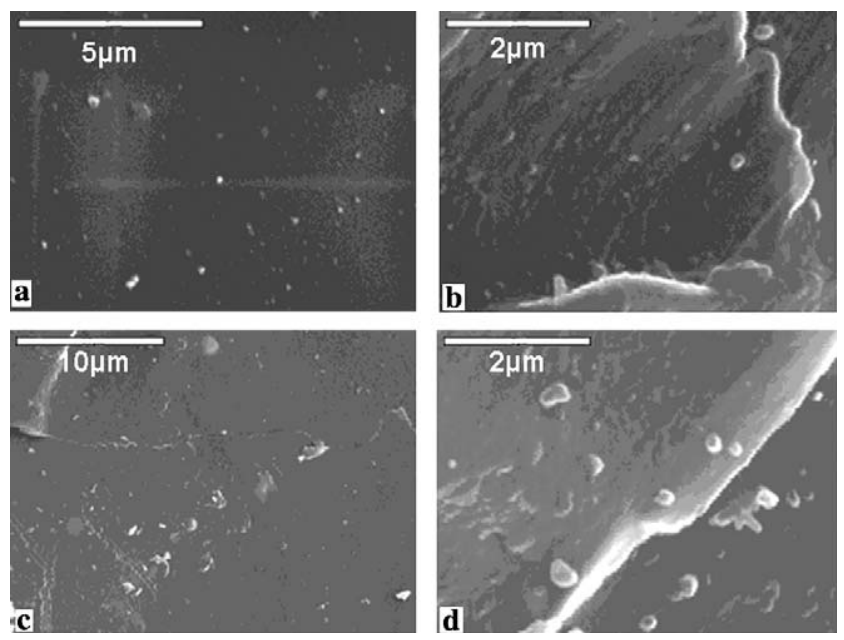

Fig. 6. Scanning electron micrographs of FEL:PVP nanodispersions taken with secondary electrons for FEL:PVP systems of (a) 10:90, (b) 25:75, (c) 50:50 and (d) 60:40 (w/w). 
Table 2

Range of martin diameters, surface mean diameters, $d_{32}$, and specific surface, $S_{\mathrm{u}}$, of particle size evaluation of investigated FEL:PVP systems

\begin{tabular}{llrc}
\hline FEL:PVP (w/w) & $\begin{array}{l}\text { Martin diameter } \\
\text { range }(\mathrm{nm})\end{array}$ & $d_{32}(\mathrm{~nm})$ & $S_{\mathrm{u}}\left(\mathrm{nm}^{-1}\right)$ \\
\hline $100: 0$ & $20,000-100,000$ & 29.18 & 0.2 \\
$75: 25$ & $250-2500$ & 17.67 & 3.4 \\
$50: 50$ & $200-500$ & 1.01 & 5.9 \\
$40: 60$ & $110-280$ & 0.29 & 21.0 \\
$25: 75$ & $50-130$ & 0.15 & 39.0 \\
$10: 90$ & $30-100$ & 0.14 & 44.0 \\
\hline
\end{tabular}

The characteristic parameters for particle size evaluation are presented in Table 2. It is observed that increasing the concentration of the polymer in the system the particle size value is decreased.

In order to further investigate the effect of polymer concentration on the particle size parameters, the correlation between the polymer concentrations in the matrix relative to the specific surface $\left(S_{\mathrm{u}}\right)$ was evaluated as presented in Fig. 7. It is observed that the alteration of the $S_{\mathrm{u}}$, as a representative factor of particle size, follows similar pattern with the intensity of interactions measured by UV spectroscopy (Fig. 4). For polymer concentration over $50 \%(\mathrm{w} / \mathrm{w})$ an impressive increase of the slope is observed similar to the interaction (Fig. 4). This intense modification of particle size and specific surface of the FEL nanoparticles cannot be attributed only to the polymer properties as the fitting is not linear to the polymer concentration. Furthermore, the high correlation with the curve (Fig. 4), that represents the intense of interactions, leads to the conclusion that these interactions correspond to the basic mechanism that is responsible for the impressive particle size reduction and specific surface increase of the drug particles. This result was expected as the development of strong heteromolecular forces normally inhibits agglomeration and recrystallization leading to optimal dispersion of the sys-

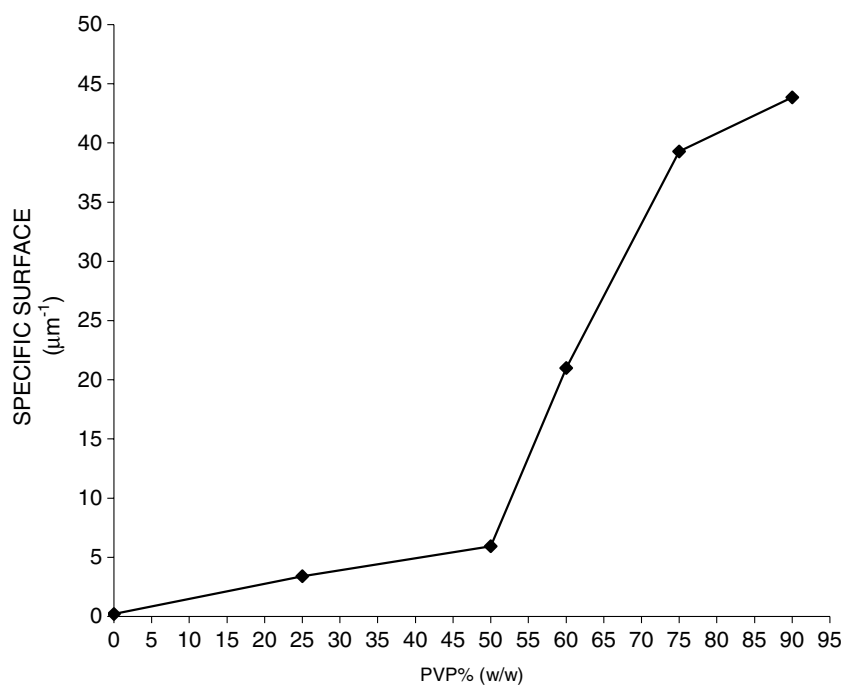

Fig. 7. Specific surface $\left(S_{\mathrm{u}}\right)$ of FEL particles versus PVP concentrations in the system. tem. It has to be referred that the specific surface behaviour in the systems also exhibits a degree of correlation with the solubility behaviour shown in Fig. 5, as the slope increase in the solubility curve corresponds to the polymer concentration area where the $S_{\mathrm{u}}$ is optimal (PVP concentration over $75 \%(\mathrm{w} / \mathrm{w}))$.

There is a consideration if the interactions affect the solubility directly or through the mechanism of particle size reduction as mentioned by Kerč [14]. As the solubility is a physicochemical constant, it cannot be modified by only the particle size reduction. The particle size reduction could increase the rate of dissolution but not the solubility itself. We believe that the particle size modification could affect the dissolution as a secondary factor, but the solubility increase has to be attributed to a direct effect of the interaction's intensity on that parameter.

\subsection{Microscopic evaluation of the dissolution behaviour}

In the previous experiments it was found that the presence of interactions in the solid state strongly affects the particle size distribution and the solubility of the nanodispersions. However, the main question "what happens after the contact of the system with aqueous media" remains. Taking into account that FEL is a hydrophobic insoluble compound there are two possible risks. Firstly, there is a possibility of poor water penetration in the microtablets, which could inhibit the availability of FEL nanoparticles. Secondly, there and their contact with the dissolution media is a high risk of direct recrystallization or agglomeration of FEL during its contact with the aqueous media.

As far as the penetration capabilities concerned, it was observed during the experiment that no water penetration took place for the microtablets composed by pure FEL, even though it remained in the aqueous solution up to $2 \mathrm{~h}$. On the contrary, the microtablets composed by the FEL:PVP systems of 25:75, and 10:90 (w/w) correspond to 15 and $9 \mathrm{~min}$, respectively, for their total dissolution.

It is obvious that the hydrophilicity of PVP plays a critical role in the optimization of water penetration. Nevertheless, it is questionable if the presence of interactions could also affect the water penetration behaviour. For this reason, a comparative evaluation of the FEL:PVP nanodispersion system of 10:90 (w/w) was performed with the relevant physical mixture. It was found that the physical mixture appeared to have a slower penetration corresponding to $18 \mathrm{~min}$ for total dissolution instead of the 9 min corresponding to the nanodispersion system. A characteristic microphotograph of the two systems is shown in Fig. 8.

It is observed that the physical mixture appears to have big particles which could be crystals or agglomerates. On the contrary, the nanodispersion system corresponds to a fine dispersion in the aqueous layer, comparable to the solid systems. As no particle discrimination could be achieved up to a magnification of $100 \times$ it could be concluded that the particle size of FEL in the aqueous phase is less than 

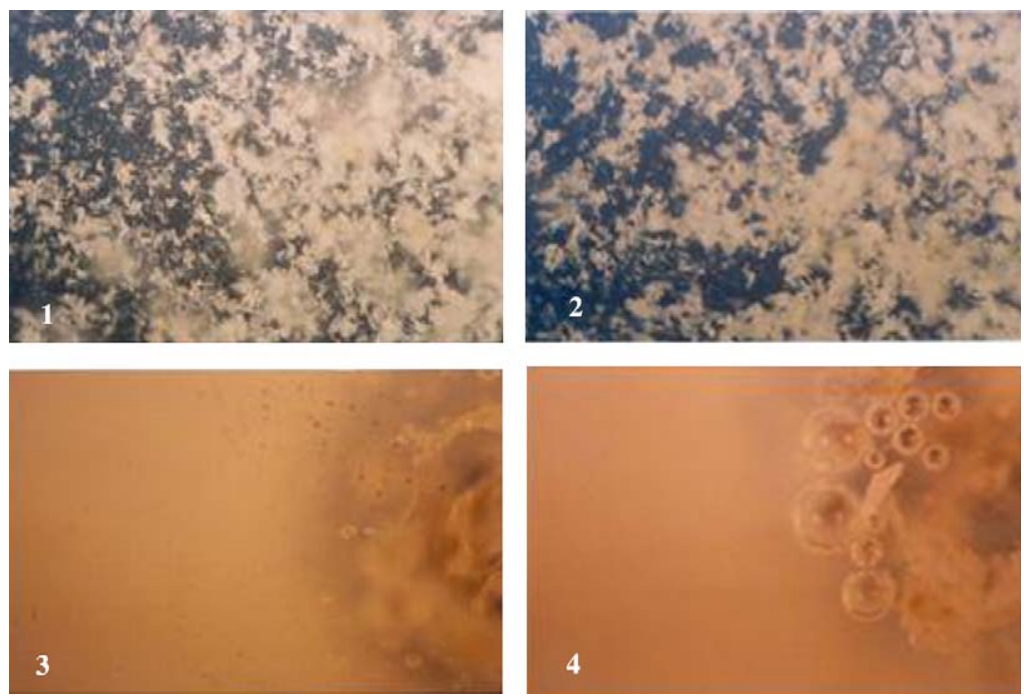

Fig. 8. Appearance of the dissolution of the physical system (up) and nanodispersion system (down) at magnifications $70 \times$ (left) and $100 \times$ (right) for the FEL:PVP systems of 10:90 (w/w).

$2.5 \mu \mathrm{m}$, with a high possibility of colloidal dispersion. Taking under consideration that the discriminative capability of the necked eye is about $250 \mu \mathrm{m}$, the above findings agree with Serajuddin's approach [26], describing that the solid dispersion systems of poorly soluble drugs appear as a colloidal dispersion after disintegration while for the conventional tablets or capsules the particles are much larger. The same researcher, in a later article [2], claims that in solid dispersions, a portion of the drug dissolves immediately, to saturate the gastrointestinal fluid, and the excess drug precipitates out as fine colloidal particles, while in conventional forms the dissolution rate is limited by the size of the primary particles formed after the disintegration which usually varies between 5 and $100 \mu \mathrm{m}$.

Conclusively, it could be said that the treatment with PVP and the presence of interaction have a significant effect on the dissolution mechanism and it is expected to have a significant effect on the release profile. The findings of this experiment also verify the hypothesis described in the relevant paragraph of solubility investigation corresponding to the creation of a complex between FEL and PVP, in aqueous media, based on the development of a hydrogen bond.

\subsection{Contact angle}

All the previous experiments showed an impressive enhancement of the hydrophilic properties of FEL in the nanodispersions with a polymer concentration more than $50 \%(w / w)$. Such enhancement seems to be strongly correlated with the development of hydrogen bonding interactions between the polymer and the drug substance.

Nevertheless, there is a concern if that optimization is indeed caused by the presence of interactions or the high concentration of the polymer modifies the system by changing the total hydrophilicity. It has been shown by York [27] that the hydrophobic character of mixtures between hydrophilic excipients (Lactose, 5 tarch) and hydrophobic drug substance (Phenobarbital) is eliminated for excipient concentrations more than $50 \%(\mathrm{w} / \mathrm{w})$, while the solubility of the systems is increased. For these investigations the polar factor and the surface polarity, as proposed by Zograffi and Tam [28], were used. The determination of contact angle was selected for this investigation in the present study as it is a representative factor for the surface polarity and hydrophilicity of the systems. Contact angle has also been used for the provision of the mechanical properties of pharmaceutical powders. The method of direct measurement of the contact angle was used (Fig. 9) as the most suitable for the investigated systems [29].

The correlation between PVP concentration and contact angle is shown in Fig. 10. Although, all the other parameters investigated in this study are impressively modified for PVP concentration more than $50 \%$ this does not happen for the contact angle. The function is highly correlated $\left(R^{2}=0.97\right)$ with a linear regression. The absence of any change of the slope and the linear fitting of the function is a verification that the impressive enhancement of the
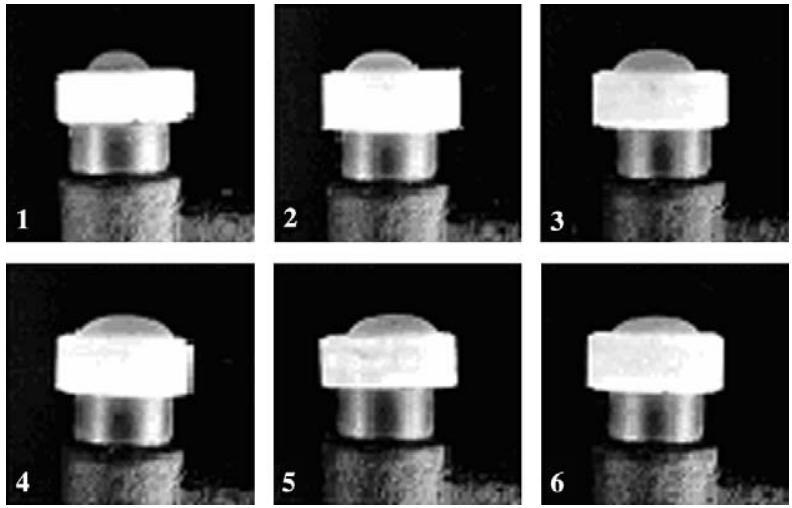

Fig. 9. Contact angle for the FEL:PVP system of: (1) 100:0, (2) 75:25, (3) 50:50, (4) 25:75, (5) 15:85 and (6) 10:90 (w/w). 


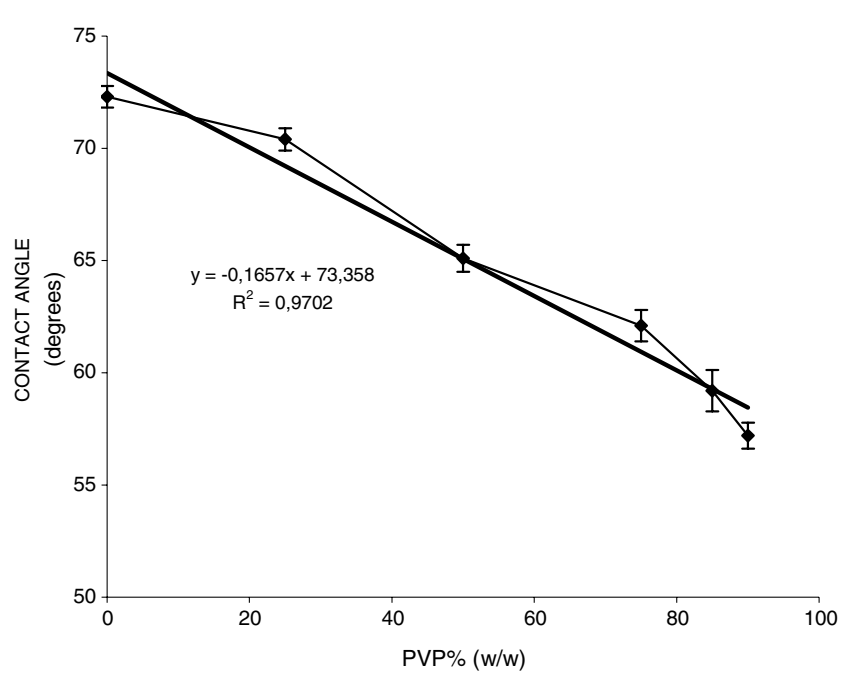

Fig. 10. The contact angle in the FEL:PVP systems versus PVP concentration in the system.

hydrophilicity of the systems for polymer concentrations over $50 \%$ is not caused on the hydrophilic properties of the polymer as found by York for other systems, but in our system that modification is for sure due to the effect of the hydrogen bonding interactions.

\subsection{Release kinetics}

As the aim of the present study is mainly the investigation of the effect of hydrogen bonding interactions on the dissolution profile of FEL. The release kinetics of several solid dispersion systems were evaluated as presented in Fig. 11 compared to that of pure FEL. The dissolution method used in this study is based on the method used by Wingstrand et al. [30] and Abrahamsson et al. [31].

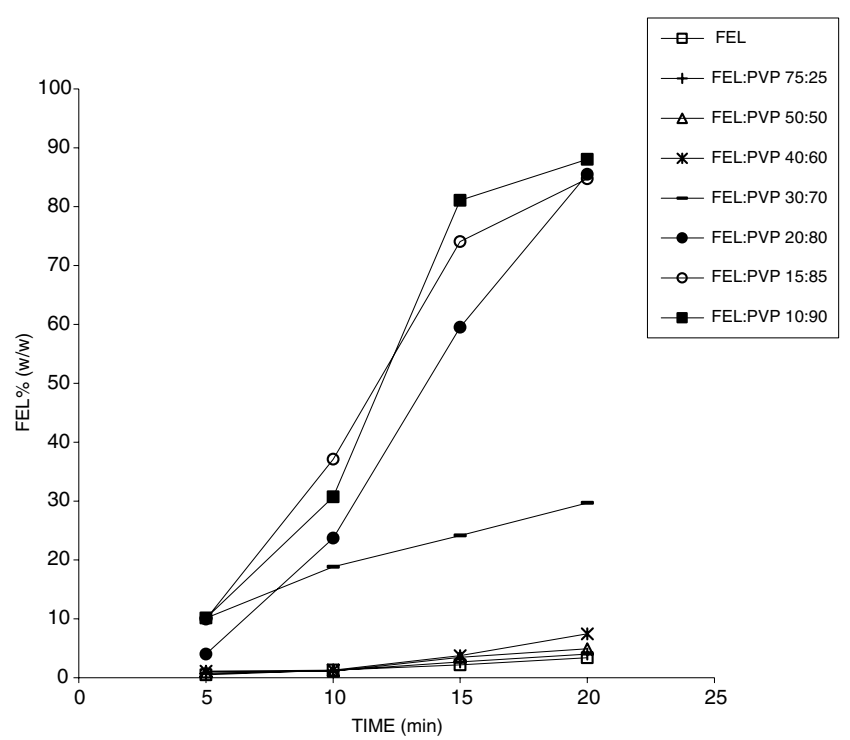

Fig. 11. Dissolved FEL\% (w/w) versus time of the pure FEL and FEL:PVP systems.
The above investigators proceeded also to in vitro in vivo' correlation of the method for several formulations of FEL with several hydrophilic matrices. The results were presented as weight per cent dissolved amounts referred to the total amount of each sample, which corresponds to $10 \mathrm{mg}$ of FEL. As the results are normalized as a percentage of the same initial amount of FEL, the Area under the curve (AUC) was selected as a representative factor for the comparison of the different systems, as it is capable of presenting the same information with the Dissolution Efficiency which is widely used.

A correlation between dissolution AUC for 20 min and the concentration of PVP in the systems is shown in Fig. 12. The AUC was calculated by using the trapezoidal rule. As shown in Figs. 11 and 12 no significant modification of FEL release kinetics is observed for polymer concentrations up to $60 \%(\mathrm{w} / \mathrm{w})$. On the contrary an impressive increase of the slope is observed for PVP concentrations over $60 \%(\mathrm{w} / \mathrm{w})$ following a linear increase.

It is observed that the systems appear to have two distinct behaviours for polymer concentrations less and more than $60 \%(\mathrm{w} / \mathrm{w})$. As the systems are composed by the same materials (FEL-PVP) and the method of preparation is identical, a linear regression could be expected in the case that the dissolution behaviour would be affected only by the drug:polymer ratio.

According to the approaches given by Lloyd et al. [11] and Sjokvist and Nystrom [12] the release mechanism could be characterized as "drug controlled "up to a polymer concentration of $60 \%(\mathrm{w} / \mathrm{w})$, while the mechanism is modified to "carrier controlled" for PVP concentration over $60 \%(\mathrm{w} / \mathrm{w})$. As the release mechanism cannot be classified according to the available by the literature approaches, the impressive deviation from the expected behaviour has to be attributed to other causes.

Comparing the results presented in Fig. 12 with the findings of this study concerning the intense of interactions

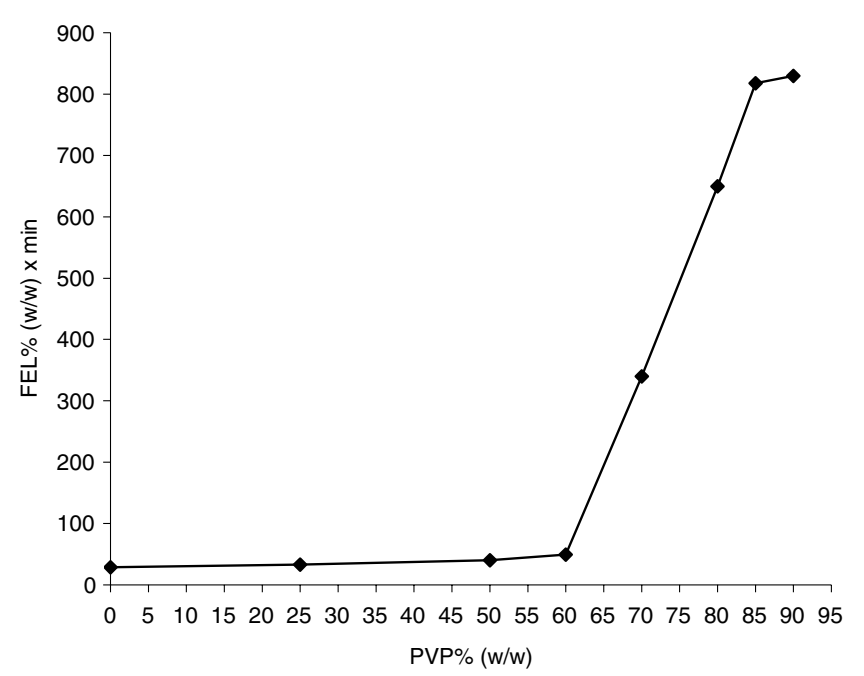

Fig. 12. The area under curve (AUC) versus PVP concentration of FEL:PVP solid dispersion systems. 
(NMR, UV), it is observed that the modification of the dissolution kinetics is observed in the area of PVP concentrations where the hydrogen bonding interactions are intense. So it can be concluded that the hydrogen bonding interactions are the cause for the dissolution enhancement of FEL from FEL:PVP binary systems for high polymer concentrations. The mechanism of such enhancement could be attributed to the effect of the interactions on the solubility and the specific surface of FEL particles in the system as shown by the comparison of the above factors, as presented in Figs. 5 and 7, with Fig. 12.

\subsection{Dynamic particle size evaluation during dissolution}

The dissolution rate of the drug in aqueous dissolution mediums is influenced by the interactions evolved between the drug particles or molecules and the macromolecular chains of the polymer matrix. These interactions can promote the dispersion of the drug that is surrounded, in molecular level, by polymer chains, and restrict its ability to form crystal lattices with its neighbouring drug molecules. It is crucial to recognize that, the reduction of the particle size is one of the most important factors which can enhance its dissolution rate, when dealing with poorly water soluble drugs. However, such a reduction of particle size usually increases the surface energy of the dispersed particles and thus may cause their agglomeration. It constitutes a negative progress, since the available surface would reduce again and possibly result in a decrease of the dissolution rate of the drug. Furthermore, the entire attempt seems to converge to the fact that the preparation of amorphous stages inhibited the drug crystallization. Dissolution studies of several micronized drugs have shown that when the drug concentration in the interactive mixtures is increased, the dissolution rate decreases, mainly due to the agglomeration process that takes place [32-34]. However, modelling of the dissolution data showed that dissolution of drugs occurred from dispersed drug particles and agglomerates [35]. For this reason in the present study, in order to understand the mechanism of dissolution, it is essential to correlate the dissolution rates of different solid dispersions with the formation of such agglomerates during dissolution.

In Fig. 13 the intensity of the particle size distribution of PVP nanoparticles during dissolution for several times is presented, compared with the corresponding values for the PVP/FEL system of $90 / 10(\mathrm{w} / \mathrm{w})$. The bigger particle size in PVP/FEL system is attributed to the incorporation of FEL in PVP and to the formation of agglomerates.

In the case of PVP, both the intensity and the average particle size diameter remain stable after the immersion of the polymer in water (Fig. 13a). In the solid dispersion system of PVP/FEL of $90 / 10(\mathrm{w} / \mathrm{w})$ the intensity reduces and the average particle diameter increases after the immersion of the dispersion system in water (Fig. 13b). It is attributed to the pressing out of the small particles during dissolution and also to the formation of agglomerates
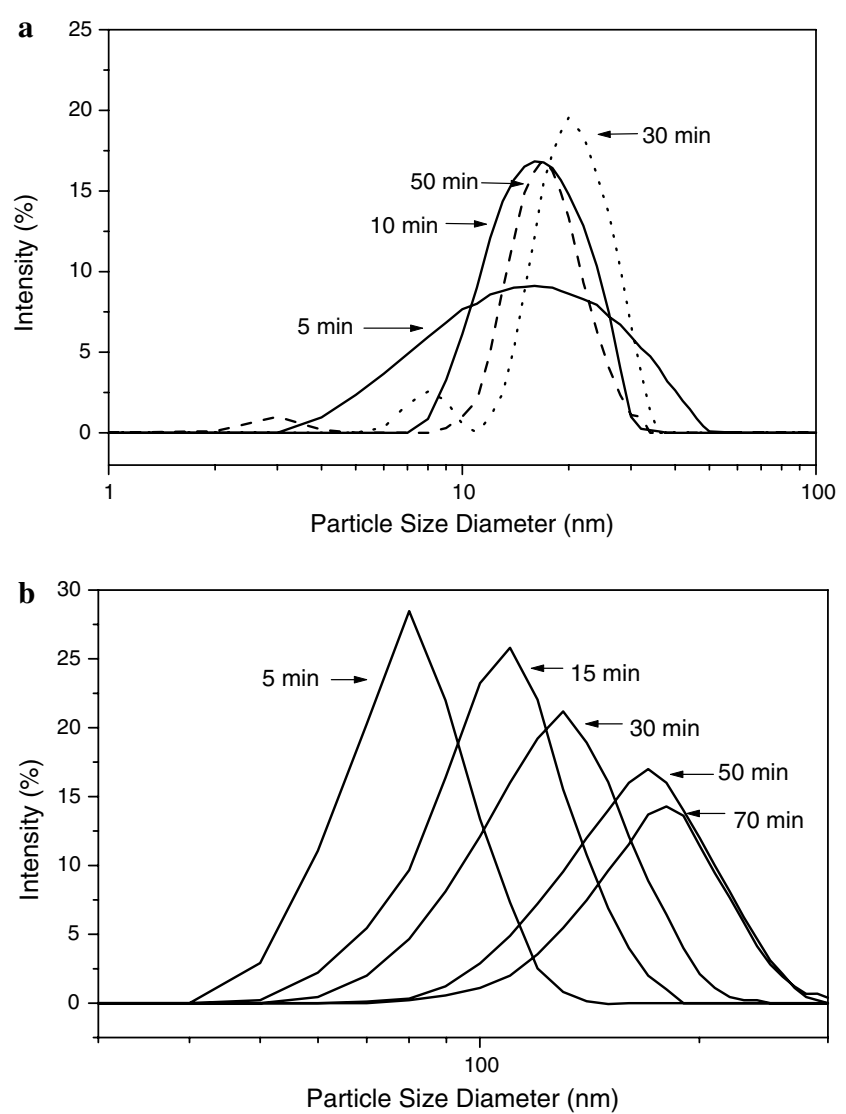

Fig. 13. Particle size distribution of: (a) PVP and (b) PVP/FEL system of $90 / 10(\mathrm{w} / \mathrm{w})$ for several dissolution times.

with a particle size up to $180 \mathrm{~nm}$. For the dispersion system of PVP/FEL of $80 / 20(\mathrm{w} / \mathrm{w})$ a particle size up to $400 \mathrm{~nm}$ was found.

It should be outlined that FEL is a highly permeable drug throughout the gastrointestinal tract. This ensures sink conditions during the dissolution of FEL from its preparations in vivo. Therefore, it is unlikely for large agglomerates as the previously mentioned to be formed in the gastrointestinal fluids. In conclusion, FEL is released from its solid dispersion in the range of nanoparticles, while it is rapidly absorbed. This combination can explain the increased bioavailability of the solid dispersions, as was found from our unpublished data.

\section{Conclusion}

The presence of hydrogen bonding interactions in FEL:PVP solid dispersion systems is the main cause for the dissolution enhancement of FEL. The basic mechanism is the effect of those interactions on the particle size distribution and the solubility of FEL. The intensity of interactions modifies the dissolution mechanism from "carrier controlled" to "drug controlled" for high polymer concentrations while the hydrophilic properties of PVP have no significant effect on this impressive enhancement. 


\section{Acknowledgement}

This study was supported by the Pharmaceutical Company, Pharmathen S.A., 6 Dervenakion Str., P.C. 15351 Pallini Attikis, Greece.

\section{References}

[1] D.-A. Wadke, A.-T.-M. Serajuddin, H. Jacobson, Preformulation testing, in: H.-A. Lieberman, L. Lachman, J.-B. Schwartz, Marchel Dekker (Eds.), Pharmaceutical Dosage Forms: Tablets, vol. 1, New York, 1989, pp. 1-73.

[2] A.-T.-M. Serajuddin, Solid dispersion of poorly water-soluble drugs: early promises, subsequent problems and recent breakthroughs, J. Pharm. Sci. 88 (1999) 1058-1066.

[3] K. Sekiguchi, N. Obi, Studies on absorption of eutectic mixture. I. A comparison of the behaviour of eutectic mixture of sulfathiazole and that of ordinary sultathiazole in man, Chem. Pharm. Bull. 9 (1961) 866-872.

[4] D.-Q.-M. Craig, The mechanisms of drug release from solid dispersions in water-soluble polymers, Int. J. Pharm. 231 (2002) 131-144.

[5] E. Karavas, G. Ktistis, A. Xenakis, E. Georgarakis, Miscibility behaviour and formation mechanism of stabilized felodipine-polivinylpyrrolidone amorphous solid dispersions, Drug Dev. Ind. Pharm. 31 (2005) 473-489.

[6] E. Karavas, E. Georgarakis, B. Bikiaris, T. Thomas, V. Katsos, A. Xenakis, Hydrophilic matrices as carriers in felodipine solid dispersion systems, Progr. Colloid Polym. Sci. 118 (2001) 149-152.

[7] W.-L. Chiou, S. Riegelman, Pharmaceutical applications of solid dispersions, J. Pharm. Sci. 60 (1971) 1281-1302.

[8] O.-I. Corrigan, Mechanisms of dissolution of fast release solid dispersions, Drug Dev. Ind. Pharm. 11 (1985) 697-724.

[9] O.-I. Corrigan, Retardation of polymeric carrier dissolution of solid dispersions containing polyethylene glycols, Drug Dev. Ind. Pharm. 12 (1986) 1777-1793.

[10] J.-L. Dubois, J.-L. Ford, Similarities in the release rates of different drugs from polyethylene glycol 6000 dispersions, J. Pharm. Pharmacol. 53 (1985) 57-66.

[11] G.-R. Lloyd, D.-Q.-M. Craig, A. Smith, A calorimetric investigation into the interaction between paracetanol and polyethylene glycol 4000 in physical mixes and solid dispersions, Eur. J. Pharm. Biopharm. 48 (1999) 59-65.

[12] E. Sjokvist, C. Nystrom, Physicochemical aspects of drug release V1. Drug dissolution from solid particulate dispersions and the importance of carrier and drug particle properties, Int. J. Pharm. 69 (1991) 53-62.

[13] D. Bikiaris, J. Prinos, M. Botev, C. Betchev, C. Panayiotou, Blends of polymers with similar glass transition. Temperatures: a DMTA and DSC study, J. Appl. Polym. Sci. 93 (2004) 726-735.

[14] J. Kerč, S. Scric, Z. Knez, P. Sencar-Bozic, Micronization of drugs using supercritical carbon dioxide, Int. J. Pharm. 182 (1999) 33-39.

[15] T. Hosono, S. Tsuchiya, H. Matsumura, Model of interaction of amaline with polyvinylpyrrolidon, J. Pharm. Sci. 69 (1980) 824-826.

[16] G. Van den Mooter, M. Wuyts, N. Blaton, R. Busson, P. Grobet, P. Augustijus, R. Kinget, Physical stabilization of amorphous ketoconazole in solid dispersions with polyvinylpyrrolidone k25. European dispersions with polyvinylpyrrolidone k25, Eur. J. Pharm. Sci. 12 (2001) 261-269.
[17] M. Valero, I. Perez-Revuelta Blanca, L. Rodriguez, Effect of PVP $\mathrm{k}-25$ on the formation of the naproxen B-ciclodextrin complex, Int. J. Pharm. 253 (2003) 97-110.

[18] T. Matsumoto, G. Zografi, Physical properties of solid molecular dispersions of indomethacin with poly(vinyl-pyrrolidone) and poly(vinylpyrrolidone-co-vinylacetate) in relation to indomethacin crystallization, Pharm. Res. 16 (1999) 1722-1728.

[19] M.-M. Velazquez, M. Valero, L.-J. Rodriguez, S.-M.-B. Costa, M.-A. Santos, Hydrogen bonding in a non-steroidal anti-inflammatory drug naproxen, J. Photochem. Photochem. B: Biol. 29 (1995) 23-31.

[20] T. Higuchi, K.-A. Connors, Phase-solubility techniques, Adv. Anal. Chem. Instrum. 4 (1965) 117-210.

[21] S. Sethia, E. Squillante, Solid dispersion of carbomazepine in PVP k30 by conventional solvent evaporation and supercritical methods, Int. J. Pharm. 272 (2004) 1-10.

[22] P. Mura, T.-M. Faucci, G.-P. Bettinetti, The influence of polyvinylpyrrolidone on naproxen complexation with hydroxypropyl-B-cyclodextrin, Eur. J. Pharm. Sci. 13 (2001) 187-194.

[23] G.-P. Bettineti, P. Mura, Dissolution properties of Naproxen in combinations with polyvilylyrrolidone, Drug Dev. Ind. Pharm. 20 (1994) 1353-1366.

[24] P. Mura, N. Zerrouk, N. Mennini, F. Moestreli, C. Chemtob, Development and characterization of naproxen-chitosan solid systems with improved drug dissolution properties, Eur. J. Pharm. Sci. 19 (2003) 67-75.

[25] K. Daniel-Mwambete, S. Torrado, C. Cuesta-Bandera, F. PonceGordo, J.-J. Torrado, The effect of solubilization on the oral bioavailability of three benzimidazole carbamate drugs, Int. J. Pharm. 272 (2004) 29-36.

[26] A.-T.-M. Serajuddin, Bioavailability enhancement of poorly watersoluble drugs by solid dispersion in surface active and self-emulsifying vehicles, Bull. Tech. Gattefosse 90 (1997) 43-50.

[27] P. York, Studies of the effect of powder moisture content on drug release from hard gelatine capsules, Drug Rev. Ind. Pharm. 6 (1980) 605-627.

[28] G. Zograffi, S.-S. Tam, Wettability of pharmaceutical solids:estimates of solid surface polarity, J. Pharm. Sci. 65 (1976) 1145-1149.

[29] J.-T. Fell, E. Efebtakis, Contact angle determination on pharmaceutical powders: a comparison of two methods, Int. J. Pharm. 4 (1979) $153-155$.

[30] K. Wingstrand, B. Abrahamson, B. Edgar, Bioavailability from felodipine extended-release tablets with different dissolution properties, Int. J. Pharm. 60 (1990) 151-156.

[31] B. Abrahamsson, D. Johansson, A. Torstensson, K. Wingstrand, Evaluation of solubilizers in the drug release of hydrophilic matrix extended-release tablets of felodipine, Pharm. Res. 11 (1994) 1093-1097.

[32] P.J. Stewart, B. Always, Aggregation during the dissolution of diazepam in interactive mixtures, Particulate Sci. Technol. 13 (1995) 213-226.

[33] R. Supabphol, P.J. Stewart, Aggregation during the dissolution of diazepam in interactive and granulated mixtures, Pharm. Sci. 2 (1996) 233-236.

[34] J. Liu, P.J. Stewart, Deaggregation during the dissolution of benzodiazepines in interactive mixtures, J. Pharm. Sci. 87 (1998) 1632-1638.

[35] B. Alway, R. Sangchantra, P.J. Stewart, Modelling the dissolution of diazepam in lactose interactive mixtures, Int. J. Pharm. 130 (1996) 213-224. 